\title{
O PROCESSO DE ENFERMAGEM NA ATENÇÃO BÁSICA DE UM MUNICÍPIO DE ALAGOAS, BRASIL
}

\section{THE NURSING PROCESS IN THE PRIMARY ATTENTION OF A MUNICIPALITY OF ALAGOAS, BRAZIL}

\section{EL PROCESO DE ENFERMERÍA EN LA ATENCIÓN PRIMARIA DE UN MUNICIPIO DE ALAGOAS, BRASIL}

\author{
Aline Santos Costa $^{1}$, Renise Bastos Farias Dias ${ }^{2}$, José César de Oliveira Cerqueira ${ }^{3}$, Rita de \\ Cássia Batista de Oliveira Peixoto ${ }^{4}$
}

\begin{abstract}
RESUMO
Objetivo: Analisar a aplicação do Processo de Enfermagem por enfermeiros que atuam na atenção básica de um município do agreste alagoano. Método: Trata-se de um estudo descritivo de abordagem qualitativa, com base na Análise de Conteúdo de Bardin. Foram entrevistados 14 enfermeiros e os dados qualitativos foram agrupados pela tendência de conteúdos. Resultado: As respostas agrupadas e organizadas originaram duas categorias: (1) O uso da SAE pelo enfermeiro na Atenção Básica em Saúde: potencialidades e limitações; (2) Aplicação das etapas do Processo de Enfermagem: parcialidade e desconhecimento. Conclusão: Os resultados revelaram a parcialidade na aplicação do Processo de Enfermagem no dia a dia do enfermeiro e a necessidade de empoderamento do enfermeiro quanto à sua identidade e prática profissional.
\end{abstract}

Descritores: Processo de Enfermagem; Atenção Primária a Saúde; Enfermeiro.

\begin{abstract}
Objective: To analyze the application of the Nursing Process by nurses who work in the basic care of a municipality in the rural area of Alagoas. Methods: This is a qualitative descriptive study, based on the Bardin Content Analysis. Fourteen nurses were interviewed and the qualitative data were grouped by the content trend. Results: The grouped and organized responses gave rise to two categories: (1) The use of SAE by the nurse in Primary Health Care: potentialities and limitations; (2) Application of the stages of the Nursing Process: bias and lack of knowledge. Conclusions: The results revealed the bias in the application of the Nursing Process in the daily routine of nurses and the need for nurses' empowerment regarding their professional identity and practice.
\end{abstract}

Keywords: Nursing Process; Primary Health, Nurse.

\section{RESUMEN}

Objetivo: Analizar la aplicación del Proceso de Enfermería por enfermeros que actúan en la atención básica de un municipio del agreste alagoano. Métodos: Se trata de un estudio

\footnotetext{
${ }^{1}$ Enfermeira. Especialista em Estratégia Saúde da Família. Programa de Residência em Enfermagem em Saúde Coletiva da Universidade Federal Fluminense. E-mail: asc.alinecosta@gmail.com.

${ }^{2}$ Enfermeira. Mestre em Desenvolvimento e Meio Ambiente. Docente da Universidade Federal de Alagoas, Campus Arapiraca. E-mail: renise.dias@arapiraca.ufal.br.

${ }^{3}$ Enfermeiro. Mestre em Enfermagem. Docente da Universidade Federal de Alagoas, Campus Arapiraca. E-mail: jcocerqueira@yahoo.com.br

${ }^{4}$ Enfermeira. Especialista em Obstetrícia. Mestre em Enfermagem. Docente da Universidade Federal de Alagoas, Campus Arapiraca. E-mail: rita.peixoto@arapiraca.ufal.br.
} 
descriptivo de enfoque cualitativo, basado en el análisis de contenido de Bardin. Se entrevistaron a 14 enfermeros y los datos cualitativos fueron agrupados por la tendencia de contenidos. Resultados: La respuesta se originaron agruparon y ensamblaron dos categorías: (1) El uso de SAE por enfermeras en Primaria de la Salud: potencial y limitaciones; (2) Aplicación de las etapas del proceso de enfermería: parcialidad y desconocimiento. Conclusiones: Los resultados revelaron la parcialidad en la aplicación del Proceso de Enfermería en el día a día del enfermero y la necesidad de empoderamiento del enfermero en cuanto a su identidad y práctica profesional.

Descriptores: Proceso de Enfermería; Atención Primaria de Salud; Enfermero.

\section{INTRODUÇÃO}

O Processo de Enfermagem (PE) visa à assistência ao ser humano, pressupondo a ideia de adoção de medidas específicas para a prestação de cuidados, de forma sistematizada, baseada em evidências científicas, fundamentada no conjunto de conhecimentos técnicos e científicos da área da enfermagem. ${ }^{1} \mathrm{~A}$ operacionalização do PE é possível por meio da implementação da Sistematização da Assistência de Enfermagem (SAE), que corresponde a organização do processo de trabalho da equipe de enfermagem.

O PE possui cinco etapas distintas: coleta de dados, diagnóstico de enfermagem, planejamento de enfermagem, implementação e avaliação de enfermagem. As etapas são interrelacionadas e direcionadas às necessidades humanas e sociais da clientela. Para aplicá-las se faz necessário "um trabalho profissional particular, que demanda habilidades e capacidades cognitivas (pensamento, raciocínio), psicomotoras (físicas) e afetivas (emoções, sentimentos e valores)". 2
É preciso destacar que o PE está regulamentado pela Lei do Exercício Profissional $\mathrm{n}^{\text {o }} 7.498 / 1986$ e pela Resolução do Conselho Federal de Enfermagem (COFEN) no 358/2009 que dispõe da SAE. Esta resolução trata da obrigatoriedade da implementação da SAE nas instituições que oferecem a assistência de enfermagem, incluindo a Atenção Básica de Saúde (ABS), além de salientar que todo o processo deve ser devidamente documentado, ou seja, registrado, para que também possa servir de base científica. ${ }^{3}$

Porém, em geral, esta prática vem sendo reproduzida pelos enfermeiros de forma desarticulada com a realidade, visto este ser um sistema em processo de consolidação, no qual são imprescindíveis a vontade política, envolvimento institucional e melhoria das condições de trabalho. ${ }^{1 ; 2 ; 4}$

Isto posto, considerando ainda a expressiva participação do enfermeiro na ABS, o objetivo desse estudo foi analisar a aplicação do PE por enfermeiros que atuam na atenção básica de um município do agreste alagoano. 


\section{MÉTODO}

Trata-se de um estudo descritivo de abordagem qualitativa, realizado em Unidades Saúde da Família do município de Arapiraca, Alagoas, Brasil.

Os sujeitos da pesquisa selecionados foram enfermeiros atuantes em unidades da ABS sorteadas, de modo aleatório, sem reposição. Foram incluídos nesta pesquisa todos os enfermeiros, de ambos os sexos, com no mínimo 6 meses de atuação na atenção básica do município de Arapiraca, que aceitaram participar do estudo.

A composição da amostra foi realizada de forma conveniente, tomando como base a concordância do sujeito em participar do estudo até que fossem obtidas as informações necessárias para responder à pergunta de pesquisa, atingindo a saturação de respostas, o que ocorreu na $14^{\mathrm{a}}$ entrevista.

A coleta de dados foi realizada através de entrevista semiestruturada, com perguntas abertas sobre o conhecimento do enfermeiro sobre o PE e SAE na ABS, com foco na operacionalização do PE no cotidiano do seu trabalho. As entrevistas ocorreram durante os meses de maio e junho de 2013, no local de atuação dos enfermeiros, sujeitos da pesquisa, que assinaram o Termo de Consentimento Livre e Esclarecido e tiveram suas identidades preservadas, sendo identificados por ENF, seguidos por números arábicos, considerando a ordem de entrevista.

As entrevistas foram gravadas por gravador portátil e transcritas integralmente, para organização e análise dos dados, por meio da Análise de Conteúdo proposta por Bardin ${ }^{5}$, realizada em três etapas: a primeira foi obtida a partir do processo de seleção das entrevistas ou definição do conteúdo de análise; a segunda etapa definiu-se pela exploração do material e na terceira etapa foi utilizado o tratamento dos resultados inferência e interpretação. Para realizar a análise dos dados qualitativos, estes foram agrupados pela tendência de conteúdos, a partir do instrumento de coleta, dos quais se originaram duas categorias.

Esta pesquisa foi submetida ao Comitê de Ética em Pesquisa e Ensino pertencente ao Centro Universitário CESMAC e aprovado sob o número de protocolo $n^{\circ} 1581 / 2012$, como previsto na Resolução n466/2012, do Conselho Nacional de Saúde.

\section{RESULTADOS}

Dos 14 enfermeiros entrevistados, identificou-se uma média de 5,1 anos de atuação na atenção básica sendo que o tempo de prestação de serviço oscilou de $\geq$ 6 meses a $\leq 13$ anos. A partir das falas dos 
sujeitos, as respostas foram agrupadas e organizadas em duas categorias: (1) O uso da SAE pelo enfermeiro na Atenção Básica em Saúde: potencialidades e limitações; (2) Aplicação das etapas do Processo de Enfermagem: parcialidade e desconhecimento.

\section{$O$ uso da SAE pelo enfermeiro na}

\section{Atenção Básica em Saúde: potencialidades e limitações}

Quando os sujeitos da pesquisa foram motivados a falar sobre o PE no dia a dia do seu trabalho, os mesmos reconheceram, em seus discursos, a importância do PE e a carência de tornar a prática mais científica e, consequentemente, contribuir para a melhoria da assistência de enfermagem à população, conforme descrito em suas falas:

Quando você usa um método, planeja fica mais fácil você alcançar seus objetivos e avaliar sua atuação (ENF 1).

Usar a SAE melhoraria a qualidade da assistência, porque melhoraria a intervenção (ENF 14).

$O$ uso da SAE facilitaria e organizaria a assistência, melhoraria a conduta, principalmente por meio do desenvolvimento de diagnósticos (ENF 13).

Embora reconhecendo a importância da SAE no trabalho do enfermeiro, os mesmos declararam entender as suas atribuições como embaraçadoras para o desenvolvimento de uma assistência pautada no pensamento crítico e embasada numa metodologia. Os enfermeiros alegaram não ter como realizar a SAE devido à sobrecarga de atribuições, como é possível observar nas seguintes falas:

A gente tenta realizar a SAE, mas não consegue, porque a Atenção Básica tem muita demanda, não se tem tempo para realizar a SAE (ENF 3).

Grande demanda de atendimento, metas a serem alcançadas, muitas atribuições do enfermeiro, excesso de papel para preencher, muitos impressos, mais famílias do que a preconizada pelo Ministério da Saúde (ENF 8).

Outra limitação citada pelos sujeitos foi a falta de iniciativa do profissional e de auxílio dos órgãos fiscalizadores e reguladores para aplicação da SAE. As justificativas foram:

Falta conscientização dos profissionais $e$ dos gestores municipais... se a gestão cobrar o enfermeiro vai fazer (ENF 3).

Teria que vim da Secretaria, de cima pra baixo (ENF 4).

Teria de ter uma cobrança da secretaria ou do COREN (ENF 1).

A insegurança do profissional na aplicação da SAE foi citada como outra limitação para o uso da SAE pelo enfermeiro na UBS. Os sujeitos da pesquisa fizeram uma crítica ao enfoque dado a temática durante o processo de formação acadêmica, evidenciando a necessidade de modificações no processo 
ensino aprendizagem e de educação permanente, conforme descrevem nos seus relatos:

Tem profissional, se falar de SAE, não sabe pra onde pende. [...] Nós enfermeiros precisamos de uma maior capacitação da SAE e desses outros temas que a envolvem, porque não é muito aprofundada na academia (ENF 1).

Não uso a SAE. Ela seria possível através de adoção de instrumentos específicos, capacitação dos profissionais, melhoria do conteúdo que é passado na universidade (ENF 5).

Só usei a SAE na faculdade e nem lembro em qual período. Na realidade não se usa a SAE ao pé da letra. Quando a gente estuda acha fácil, mas na prática dificulta (ENF 7).

\section{Implementação das etapas do Processo}

\section{de Enfermagem: parcialidade e}

\section{desconhecimento}

Os sujeitos relataram em suas falas sobre o desafio da implementação de todas etapas do PE, evidenciando desconhecimentos sobre alguns aspectos do PE e SAE. Identificou-se que a etapa de investigação foi a única que todos os entrevistados relataram cumprir. Além disso, afirmaram não realizar o registro do PE adequadamente, conforme evidenciam as falas:

Não uso a SAE, nem registro (ENF 12).

Fazia na atenção Hospitalar porque acho que é mais adequado para o hospital mesmo. Na saúde da família não há recursos (ENF 6).

Certa parte a gente faz, mas não registra adequadamente (ENF 11).
A SAE a gente faz o tempo inteiro, todas as fases são realizadas, mesmo que não seja registrada. Talvez só o registro que não é adequado, mas também depende do profissional (ENF 1).

Ainda sobre a implementação do PE, a maioria dos entrevistados alegou realizar o diagnóstico de enfermagem. Porém, quando questionados acerca da taxonomia de diagnósticos usada, a maioria dos participantes afirmou não usar nenhuma classificação de diagnóstico. Somente foram citadas a taxonomia CIPE® e NANDA, sendo esta última a classificação que a maioria relatou ter aprendido na graduação. Alguns profissionais confundiram-se com os sistemas de classificação de patologias e programas de atendimento. $\mathrm{O}$ resultado revela o desconhecimento dos profissionais sobre os sistemas de classificação de diagnóstico, conforme é possível observar nas falas:

Não me lembro de nenhum sistema de classificação (ENF 10).

Eu usaria a NANDA porque eu estudei na faculdade (ENF 8).

Se fosse usar na atenção básica, usaria a CIPE porque é mais simples (ENF 9).

Eu uso a classificação de Manchester (ENF 2).

Quanto ao uso de teorias de enfermagem que fundamentassem a formação de diagnósticos e intervenções, apenas um enfermeiro citou utilizar a Teoria das Necessidades Humanas Básica 
de Wanda Horta, os demais participantes negaram o uso de teorias de enfermagem e confundiram as teorias com os conteúdos científicos abordados no processo de formação, conforme as falas descritas:

Às vezes eu uso Wanda Horta (ENF 9).

A gente usa a teoria sempre, faz o exame céfalo-caudal, dá o diagnóstico (ENF 2).

Sim, eu uso a teoria da enfermagem da parte preventiva (ENF 3 ).

\section{DISCUSSÃO}

Diante dos resultados pode-se observar que os enfermeiros conseguem reconhecer as potencialidades do uso da SAE na atividade laboral, porém ainda encontram limitações em executá-la. A sobrecarga de trabalho, administração ineficaz da gestão municipal, dentre outros motivos, são fatores que dificultam a assistência do enfermeiro na ABS. ${ }^{6}$

Além disso, identificou-se que alguns sujeitos não reconheceram a aplicabilidade do PE no eixo da ABS, relataram dificuldade em desenvolver o PE por não terem tido a oportunidade de adquirir conhecimento suficiente durante a graduação ou após. Mesmo sendo um conteúdo amplamente abordado na categoria, ainda apresenta um distanciamento entre a associação do conhecimento e a sua aplicação na prática do enfermeiro, fazendo com que o profissional exerça sua atividade sem refletir o seu processo de trabalho. ${ }^{7}$ Infelizmente, é comum a abordagem do PE nas instituições de ensino como método fragmentador, geocêntrico e desarticulado da prática, dificultando, assim, as habilidades de aplicabilidade das etapas do PE. ${ }^{8}$

Essa realidade expõe a carência no investimento das instituições de ensino, instituições de serviços, órgãos de classe, entre outras entidades em desenvolverem projetos de educação permanente que objetivem instrumentalizar a SAE nos espaços de atuação do enfermeiro, como forma de ampliar a ótica do profissional para um cuidado pautado na metodologia científica, assim como desenvolver o PE na ABS para qualificar o cuidado integral ao indivíduo, família e comunidade.

Destaca-se ainda, a necessidade da busca conjunta por melhores condições de trabalho, visto que a precarização do trabalho pode interferir no sucesso da prática profissional do enfermeiro. $\mathrm{O}$ enfermeiro em seu âmbito de atuação desenvolve atividades variadas, convivendo com mecanismos de poder internos e externos ao seu fazer diário, geralmente originado do modelo mecanicista e biomédico. Para maior autonomia de sua prática, o enfermeiro precisa diminuir a cisão entre o saber e o fazer enfermagem. ${ }^{9}$ 
As atribuições e ações inerentes ao fazer do enfermeiro devem ter a prerrogativa de um processo de trabalho planejado, fundamentadas no método científico, na prática baseada em evidências e estar de acordo com a Lei do Exercício Profissional no 7.498/1986 e com a Resolução COFEN no 358/2009 que dispõe da SAE. O PE precisa ser executável e avaliado, buscando cuidar com responsabilidade, desmitificando o fazer empírico, desorganizado e muitas vezes desqualificado, favorecendo uma melhor assistência ao indivíduo e coletividade em diferentes momentos do processo saúde e doença. ${ }^{10}$

A operacionalização do PE apresenta algumas dificuldades que se interpõem, a destacar: a não compreensão do enfermeiro sobre o seu papel, associado à percepção do $\mathrm{PE}$ como obrigação institucional e não como ferramenta para a qualificação do cuidado em saúde, além do quantitativo insuficiente de enfermeiros nas instituições de saúde e o excesso de atividades administrativas. $^{11}$

As limitações para aplicação do PE na unidade básica de saúde perpassaram também pela parcialidade da aplicação das fases do PE, incluindo registros de enfermagem incompletos ${ }^{12}$ e insegurança sobre o uso do sistema de classificação de diagnósticos. Quando não se executa adequadamente, o PE gera invisibilidade e falta de reconhecimento profissional, além de dificultar ao enfermeiro a avaliação da sua prática. $^{2} \mathrm{O}$ registro do $\mathrm{PE}$ é de responsabilidade do enfermeiro, está disposto na Resolução COFEN $n^{\circ}$ 429/2012 e deve ser realizado em todos os níveis da atenção à saúde das pessoas, famílias e coletividades humanas, gerando evidências sobre a eficácia e eficiência das ações/intervenções de enfermagem. ${ }^{13}$

A falta de uma aproximação maior com o tema pode explicar a pouca aplicabilidade do PE e das teorias de enfermagem. O uso crítico das teorias de enfermagem no PE contribuiria para a qualificação do cuidado. Ao contrário, a prática do enfermeiro volta-se para tarefas, atendendo às demandas do serviço e não às necessidades do paciente. $^{14}$ Assim, a assistência organizada e prestada com base nas teorias de enfermagem corrobora para a autonomia do trabalho do enfermeiro. ${ }^{9}$

O enfermeiro, apesar de ter superado barreiras e conquistado mais espaço em seu campo de atuação, ainda precisa empoderar-se da sua identidade, da sua prática científica, tornar-se responsável pela realização do PE, que é inerente à sua profissão. Compreender que o PE é algo indispensável para o exercício profissional, cujas respectivas etapas do PE são indissociáveis e indispensáveis. Essa compreensão se faz necessária mesmo que o PE não esteja plenamente consolidado 
nos ambientes em que ocorre o cuidado de Enfermagem.

\section{CONCLUSÃO}

Ainda há muitas lacunas entre a formação acadêmica e a prática profissional do enfermeiro, principalmente no tocante a implementação do método científico na atenção básica. As falas dos enfermeiros entrevistados demonstraram falta de conhecimento e pouco domínio nesta temática. Somado a isso, constatouse a fragmentação na aplicação das etapas do PE.

As dificuldades foram muitas para a não aplicação do $\mathrm{PE}$, destacando-se a sobrecarga de tarefas pelo enfermeiro, a falta de recursos materiais e humanos e a falta de apoio de órgãos de gestão e de fiscalização. De fato, estes pontos são limitações concretas para o bom desenvolvimento do trabalho do enfermeiro. Ainda há um longo caminho a se percorrer nesse campo de atuação e na busca de uma formação plena, crítica, reflexiva, competente e transformadora da realidade, para assistir a população de maneira adequada. Sobretudo, necessita-se ainda de empoderamento do enfermeiro quanto à sua identidade e prática profissional.

Nesse contexto, faz-se necessária a realização de mais estudos relacionados ao PE na atenção básica, uma vez que não foram avaliados os registros dos profissionais do estudo, nem acompanhada a rotina do trabalho do enfermeiro a fim de entender melhor suas falas, as potencialidades e fragilidades da aplicação do PE na ABS, de modo que os enfermeiros alcancem o exercício profissional integral, executado sistematicamente, baseado nos princípios do pensamento crítico, voltado para a valorização do enfermeiro.

\section{REFERÊNCIAS}

1. Garcia TR, Nóbrega MML. Processo de enfermagem: da teoria à prática assistencial e de pesquisa. Esc Anna Nery Rev Enferm. [Internet]. 2009 [citado em 21 jun 2017]; 13(1):188-93. Disponível em: http://www.redalyc.org/articulo.oa?id=127 715321025

2. Garcia TR. Sistematização da assistência de enfermagem: aspecto substantivo da prática profissional [Editorial]. Esc Anna Nery Rev Enferm. [Internet]. 2016 [citado em 24 de jan 2018]; 20(1):5-6. Disponível em:

http://www.scielo.br/pdf/ean/v20n1/14148145-ean-20-01-0005.pdf

3. Conselho Federal de Enfermagem. Resolução n ${ }^{\circ}$ 358/2009. Dispõe sobre a Sistematização da assistência de enfermagem e a implementação do processo de enfermagem em ambientes, públicos ou privados, em que ocorre o cuidado profissional de Enfermagem, e dá outras providências [Internet]. Rio de Janeiro: COFEN; 2009 [citado em 21 jun 2017]. Disponível em: http://www.cofen.gov.br/resoluo-cofen3582009_4384.html

4. Silva EGC, Oliveira VC, Neves GBC, Guimarães TMR. O conhecimento do enfermeiro sobre a sistematização da assistência de enfermagem: da teoria à 
prática. Rev Esc Enferm USP. [Internet]. 2011 [citado em 21 jun 2017]; 45(6):

1380-6. Disponível em:

http://www.scielo.br/scielo.php?script=sci _arttext\&pid=S0080-62342011000600015

5. Bardin L. Análise de conteúdo. Lisboa: Edições 70; 2000.

6. Andrade LDF, Simões ACM, Henriques AHB, Sousa ATO, Pinto MB.

Desempenho do enfermeiro em suas atividades laborais na atenção primária à saúde. Rev Enferm Atenção Saúde [Internet]. 2016 [citado em 21 jun 2017]; 5(1):51-63.Disponível em: http://seer.uftm.edu.br/revistaeletronica/ind ex.php/enfer/article/view/1749/pdf

7. Barros ALBL, Lopes JL. A legislação e a sistematização da assistência de Enfermagem. Enferm Foco (Brasília) [Internet]. 2010 [citado em 21 jun 2017]; 1(2):63-65. Disponível em http://revista.cofen.gov.br/index.php/enfer magem/article/viewFile/17/18

8. Santos MG, Bittencourt JVOV, Orlandi M, Sbardelotto T, Migliorini O, Celich KS, et al. $\mathrm{O}$ ensino do processo de enfermagem (PE) nos cursos de enfermagem em uma cidade do oeste catarinense. Rev Teor Prát Educ. [Internet]. 2015 [citado em 24 jan 2018]; 7(1):21-27. Disponível em https://www.mastereditora.com.br/periodic o/20150827_210708.pdf

9. Santos FOF, Montezeli JH, Peres AM. Autonomia profissional e sistematização da assistência de enfermagem: percepção de enfermeiros. REME Rev Min Enferm. [Internet]. 2012 [citado em 24 jan 2018]; 16(2):251-7. Disponível em: http://www.reme.org.br/artigo/detalhes/526 10. Antunes MJM, Guedes MVC. Integralidade nos processos assistenciais na atenção básica. In: Garcia TR, Egry EY, [organizadores]. Integralidade da atenção no SUS e sistematização da assistência de enfermagem. Porto Alegre: Artmed; 2010. p.19-27.

11. Braga LM, Torres LM, Ferreira VM.

Condições de trabalho e fazer em enfermagem. Rev Enferm UFJF. [Internet]. 2015 [citado em 16 jun 2017]; 1(1):55-63.
Disponível em:

http://www.ufjf.br/revistadeenfermagem/fil es/2015/05/10-Revista-de-EnfermagemC07.pdf

12. Barbosa SF, Melleiro MM, Sportello EF, Tronchin DMR, Mira VL. Qualidade dos registros de enfermagem: análise dos prontuários de usuários do Programa de Assistência Domiciliária de um hospital universitário. Mundo Saúde [Internet]. 2011 [citado em 21 jun 2017]; 35(4):395400. Disponível em: http://www.saocamilosp.br/pdf/mundo_saude/88/04_Qualidaded osregistrosdeEnfermagem.pdf 13. Conselho Federal de Enfermagem. Resolução $n^{\circ} 429 / 2012$. Dispõe sobre o registro das ações profissionais no prontuário do paciente, e em outros documentos próprios da enfermagem, independente do meio de suporte tradicional ou eletrônico [Internet]. Rio de Janeiro: CONFEN; 2012 [citado em 24 jan 2018]. Disponível em: http://www.cofen.gov.br/resoluo-cofen-n4292012_9263.html

14. Souza MFG, Santos ADB, Monteiro

AI. O processo de enfermagem na concepção de profissionais de enfermagem de um hospital de ensino. Rev Bras

Enferm. [Internet]. 2013 [citado em 21 jun 2017]; 66(2):167-73. Disponível em: http://www.scielo.br/pdf/reben/v66n2/03.p df

RECEBIDO: $30 / 06 / 2017$

APROVADO: $16 / 02 / 2018$

PUBLICADO: 07/2018 